\section{Comment \\ fonctionne un \\ récepteur couplé \\ aux protéines G ? \\ Le cas des \\ récepteurs \\ métabotropiques \\ du glutamate et \\ du GABA}

Thierry Galvez, Jean-Philippe Pin notamment les récepteurs métabotropiques des
deux principaux neurotransmetteurs (le glutamate et l'acide $\gamma$-aminobutyrique ou GABA), a révélé un mécanisme mettant en jeu des dimères de récepteurs. Cette observation n'est pas sans rappeler le mécanisme d'activation d'autres types de récepteurs membranaires tels que, par exemple, les récepteurs à activité tyrosine kinase de l'insuline. Ce mécanisme pourrait également s'appliquer à d'autres récepteurs couplés aux protéines $G$, et ouvrir de nouvelles voies d'action pharmacologique visant la régulation de ces récepteurs qui sont déjà la cible de près de la moitié des médicaments existants, quels que soient les domaines thérapeutiques considérés. <

$(\rightarrow) \mathrm{m} / \mathrm{s}$ $2000, n^{\circ} 5$, p. 644
Les récepteurs membranaires sont la cible d'informations extérieures à la cellule, portées par les moléculesmessages qu'ils reconnaissent. Leur rôle est de transmettre cette information à l'intérieur de la cellule. Pour cela, la liaison de la molécule-message à son récepteur est convertie en une première information structurale (un changement conformationnel du récepteur) se traduisant elle-même par l'ouverture d'un canal ionique ou la régulation d'activités enzymatiques intracellulaires (kinases, GTPases). Plusieurs stratégies moléculaires permettant d'intégrer et de transmettre à travers la membrane l'information contenue dans la liaison d'une molécule à son récepteur ont été sélectionnées par les processus évolutifs. Ces stratégies correspondent à autant de classes structurales de récepteurs, parmi lesquels se trouvent les récepteurs couplés aux protéines $G$

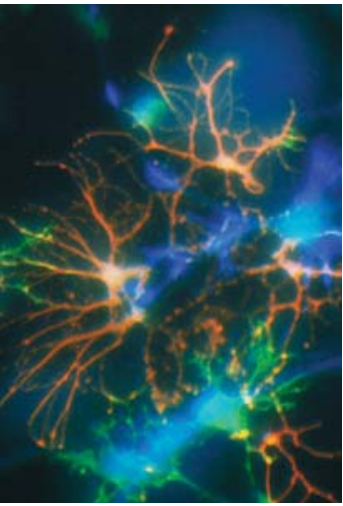

$(\mathrm{RCPG})(\rightarrow)$. Ces derniers sont les récepteurs de la majorité des hormones, des neurotransmetteurs et de certains facteurs de croissance et de différenciation cellulaire. Ils interviennent ainsi dans tous les grands systèmes de communication intercellulaire et jouent un rôle prépondérant dans la perception sensorielle chimique: ce sont en effet les récepteurs des « odeurs », des «molécules du goût », des phéromones [1]. Des gènes codant pour des RCPG ont été identifiés dans le génome d'êtres vivants très distants d'un point de vue évolutif, tels que la plante $A$. thaliana, la levure $S$. cerevisiae, le nématode $C$. elegans, la mouche $D$. melanogaster et les vertébrés, pour lesquels ils constituent une des plus grandes familles géniques. Par définition, les RCPG exercent la plupart de leurs effets cellulaires en stimulant une étape clé du cycle d'activation de GTPases hétérotrimériques (ou protéines G), l'échange GDP-GTP. Cette propriété provient de la présence, dans tous les RCPG, d'un unique motif structural, le domaine heptahélice constitué de sept 
$(\rightarrow) \mathrm{m} / \mathrm{s}$

$1998, n^{\circ} 12$,

p. 1315

$(\rightarrow) \mathrm{m} / \mathrm{s}$

$1999, n^{\circ} 11$,

p. 1211 hélices transmembranaires reliées par des boucles de longueur variable [2]. La dénomination « récepteurs heptahélices » plutôt que «RCPG » sera d'ailleurs utilisée dans la suite du texte, car si toutes les protéines de la famille des RCPG possèdent un domaine heptahélice, certaines ne semblent pas capables d'activer les protéines $G$, et d'autres sont capables d'activer des voies de signalisation différentes de celles mettant en jeu des protéines G [3].

La comparaison des structures primaires de ces régions transmembranaires permet de définir plusieurs classes de récepteurs heptahélices [4]. La plus « célèbre » est la classe des récepteurs apparentés à la rhodopsine $(\rightarrow)$, ou classe I, qui regroupe, de manière non exhaustive, les récepteurs olfactifs $(\rightarrow)$ et les récepteurs des amines biogènes. De la même façon, les récepteurs des peptides de type glucagon, sécrétine et PACAP (pituitary adenylate cyclase-activating polypeptide) forment la classe II. D'autres familles telles que celles des récepteurs VN des phéromones ou des récepteurs $\mathrm{T} 2$ du « goût acide » ont été plus récemment identifiées [5]. Il est communément admis que ces récepteurs fonctionnent comme des protéines «allostériques monomériques »: les agonistes se fixeraient totalement ou en partie au sein $\mathrm{du}$ domaine heptahélice pour en induire ou en sélectionner une conformation active capable d'activer les protéines G [1]. Pour les récepteurs heptahélices de la classe III, des données récentes, présentées ici, montrent qu'ils fonctionnent sous forme de dimères et que les interactions entre sous-unités sont essentielles à leur activité.
Figure 1. La classe III des récepteurs heptahélices. A. Arbre phylogénétique des récepteurs heptahélices de classe III. L'arbre a été construit à partir de l'alignement des séquences des domaines heptahélices uniquement [6]. On distingue plusieurs grands groupes: les récepteurs métabotropiques du glutamate (mGlu), les récepteurs métabotropiques du GABA $\left(G A B A_{B}\right)$, les récepteurs $T_{1}$ du goût, des récepteurs présomptifs des phéromones, les récepteurs-détecteurs $d u$ calcium (CaS) ainsi que des récepteurs olfactifs de certains poissons osseux. Le récepteur Bride of sevenless (BOSS) de D. melanogaster ainsi que des récepteurs orphelins clairement apparentés à cette classe de récepteurs n'ont pas été inclus dans cet arbre par souci de simplification. Des séquences issues de plusieurs espèces animales sont représentées: Homo sapiens (rouge), Rattus norvegicus et Mus musculus (bleu), les poissons Fugu rubripes et Carassius auratus (violet), Drosophila melanogaster (vert foncé), Caenorhabditis elegans (orange). Des séquences apparentées sont aussi présentes chez des organismes primitifs tels que l'éponge Geodia cydonium (noir) et l'amibe Dictyostelium discoideum (pointillé noir). B. Organisation topologique des domaines structuraux des récepteurs heptahélices de classe III. Outre un domaine transmembranaire heptahélice (bleu), la majorité de ces récepteurs présente un domaine extracellulaire (rose) appelé domaine Venus flytrap. Un domaine riche en cystéines (9 cystéines pour 80 résidus, représenté en jaune) s'intercale entre le domaine Venus Flytrap et les sept domaines transmembranaires, sauf dans le cas des récepteurs $\mathrm{GABA}_{B}$. $C$. Un récepteur de classe III conformément à la représentation schématique utilisée dans cet article. R.: récepteur; S: séquence signal; Ext: extracellulaire; Int: intracellulaire; Mb: membrane cellulaire. 


\section{Les fonctions très variées des récepteurs de classe III}

La classe III des récepteurs heptahélices rassemble plusieurs groupes de récepteurs présents dans de multiples espèces animales [6] (Figure 1A). II s'agit notamment du groupe des huit récepteurs métabotropiques $\left(\mathrm{mGlu}_{1-8}\right) \mathrm{du}$ principal neurotransmetteur excitateur du système nerveux central: le glutamate. Ces récepteurs - outre les récepteurs-canaux du glutamate - jouent un rôle essentiel dans la modulation de la transmission synaptique. Des molécules agissant spécifiquement sur ces récepteurs présentent un intérêt thérapeutique pour le traitement de la douleur, de l'anxiété, de la dépendance à certaines drogues. Au sein de la classe III, se trouvent également les récepteurs $G A B A_{B}$ qui, avec les récepteurs-canaux $G A B A_{A}$, sont des récepteurs de l'acide $\gamma$-aminobutyrique, le neurotransmetteur inhibiteur le plus répandu du système nerveux des vertébrés adultes ( $40 \%$ des neurones le synthétisent). La classe III regroupe également des récepteurs mis en jeu dans la perception chimique de l'environnement, tels que les récepteurs $T_{1}$ des papilles gustatives, impliqués dans la perception des saveurs sucrées et du glutamate (goût aussi dénommé « umami ») $(\rightarrow)$, ainsi qu'un groupe d'environ une centaine de récepteurs exprimés dans l'organe voméronasal des mammifères et susceptibles d'être des récepteurs des phéromones $[7,8](\rightarrow)$. Tous ces récepteurs sont également apparentés aux récepteurs olfactifs de certains poissons téléostéens, ainsi qu'aux récepteurs des ions $\mathrm{Ca}^{2+}(\mathrm{CaS})$, dont un des rôles physiologiques majeurs est d'être le « détecteur » de la concentration extracellulaire en ions $\mathrm{Ca}^{2+}\left([\mathrm{Ca}]_{\mathrm{e}}\right)$ au sein de la boucle de régulation de la calcémie.

\section{L'étape initiale de l'activation ou comment utiliser un « piège à mouche »}

Tous ces récepteurs de classe III présentent une même organisation structurale qui s'appuie sur deux domaines principaux (Figure IB): un domaine heptahélice, comme tous les RCPG, sur lequel est greffé un domaine amino-terminal extracellulaire de grande taille (400 à 600 résidus). Alors que pour la grande majorité des RCPG, et notamment les récepteurs de la classe de la rhodopsine, les agonistes se fixent dans le domaine heptahélice, c'est au niveau du domaine extracellulaire qu'in- $\rightarrow) \mathrm{m} / \mathrm{s}$

p. 852

$(\rightarrow) \mathrm{m} / \mathrm{s}$

1997, n²,

p. 201

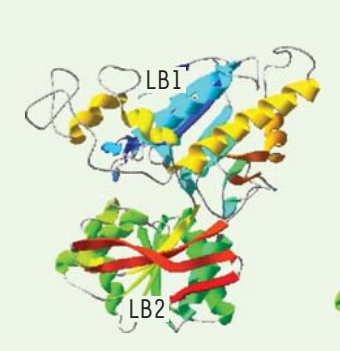

1 Conformation fermée F, vide

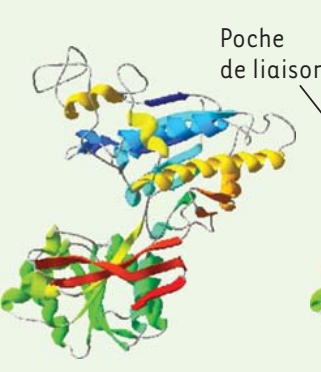

2 Conformation ouverte 0 , vide

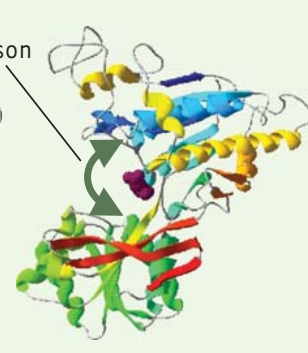

3 Conformation ouverte 0 , agoniste lié

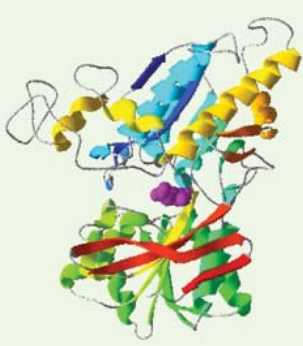

4 Conformation fermée $F$, agoniste lié

= active
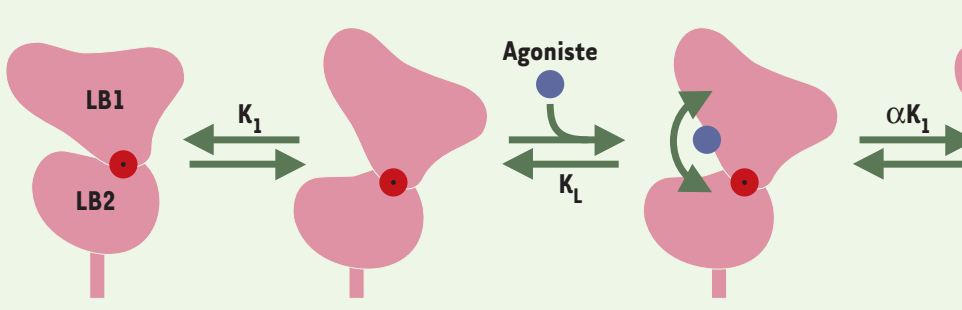

teragissent les activateurs des récepteurs de classe III. Comment la fixation d'un agoniste sur le domaine extracellulaire du récepteur peut-elle conduire à l'activation des protéines $G$ par l'intermédiaire du domaine heptahélice?

La structure du domaine extracellulaire a été récemment résolue pour le récepteur $\mathrm{mGlu}_{1}$; elle fournit quelques éléments de réponse [9]. Le domaine de liaison est un domaine globulaire constitué de deux sous-domaines, ou lobes ( $L B 1$ et LB2), entre lesquels le glutamate vient se loger (Figure 2). Chaque lobe est constitué d'un feuillet $\beta$ à sept brins, encadré sur ses deux faces par des hélices $\alpha$. Cette structure est en fait identique à celle de certaines protéines des bactéries à Gram négatif qui jouent le rôle de récepteurs des acides aminés ligand est un agoniste, la forme fermée est stabilisée $(\alpha>1)$. 
branchés lors de leur transport du périplasme vers le cytoplasme [10]. Pour toutes ces protéines, deux grands types de conformations peuvent être mis en évidence: une conformation ouverte, qui crée une large poche entre les deux lobes, et une conformation fermée, caractérisée par le rapprochement des deux lobes (Figure 2). Ces deux types de conformation sont en équilibre, mais la forme ouverte est prépondérante en l'absence de ligand, tandis que la forme fermée serait stabilisée après la fixation de l'agoniste (Figure 2). Par analogie avec le piège à mouche des plantes carnivores de la famille Dioneae (ou communément Venus flytrap en anglais), ce mécanisme a été appelé Venus flytrap. Si, dans le cas des récepteurs mGlu, le glutamate peut interagir avec une conformation du domaine de liaison ouverte ou fermée, il établit plus de liaisons avec la protéine en conformation fermée, ce qui stabilise cette dernière qui serait donc la forme active. Au contraire, la liaison des antagonistes est incompatible avec une conformation fermée du domaine de liaison, qui ne peut alors qu'adopter une conformation ouverte, inactive [11, 12].

La fermeture du domaine Venus flytrap constituerait donc une des premières étapes de l'activation d'un récepteur de classe III. Mais comment ce changement conformationnel conduit-il à l'activation du domaine heptahélice, puis à celle des protéines $G$ ?

\section{Pour communiquer, l'important est d'être deux: le rôle de la dimérisation dans le mécanisme d'activation}

Dès 1996, il a été montré que des RCPG de classe III existaient sous forme de dimères constitutifs [13]. Ces dimères peuvent être des homo- ou des hétérodimères. Les récepteurs mGlu et $\mathrm{CaS}$ sont des homodimères. La démonstration définitive en a été apportée avec la résolution de la structure du domaine extracellulaire du récepteur $\mathrm{mGlu}_{1}$, qui se révèle être un dimère de domaines Venus Flytrap [9]. La participation directe des domaines heptahélices à la cohésion et au fonctionnement de l'oligomère est attendue, mais n'est pas encore clairement démontrée.

Le récepteur $G_{A B A_{B}}$ est, quant à lui, un récepteur hétérodimérique formé par l'assemblage de deux récepteurs heptahélices appelés $G A B A_{B 1}$ et $G A B A_{B 2}$. Le récepteur $G A B A_{B}$ a été identifié sur le plan pharmacologique il y a plus de vingt ans par Bowery. II s'agit d'un type de récepteur du GABA distinct des récepteur-canaux de type $G A B A_{A}$, les seuls connus à cette époque [14]. Ce n'est qu'en 1997 que l'équipe de Bettler (Bâle, Suisse), a cloné le premier récepteur heptahélice du GABA, GABA ${ }_{B 1}$, capable de se lier à tous les ligands connus du récepteur $G A B A_{B}$, mais incapable d'activer les protéines $G$ [15]. En recherchant d'autres protéines homologues de $G_{A B A_{B 1}}$ ou capables de complémenter $G_{A B A_{B 1}}$ fonctionnellement, cinq groupes ont indé- pendamment identifié le récepteur $\mathrm{GABA}_{\mathrm{B} 2}$ [16-20]. $G_{B B A_{B 1}}$ et $G_{A B A_{B 2}}$ interagissent notamment (mais pas uniquement) via une interaction de type coiled-coil impliquant les domaines intracellulaires des deux protéines (Figure 3). L'assemblage de l'hétéromère est étroitement contrôlé dès les étapes de sa synthèse, car la sous-unité $G_{B B A_{B 1}}$ est retenue au sein du réticulum endoplasmique par une séquence de rétention (résidus RSRR adjacents au domaine coiled-coil) qui est masquée lorsque $G_{A B A_{B 2}}$ vient s'y associer [21]. Ainsi, les deux récepteurs heptahélices $G_{A B A_{B 1}}$ et $G_{B B A}$ sont toujours associés à la surface des cellules. Quels sont cependant le rôle et l'importance de la dimérisation dans le mécanisme d'activation des récepteurs heptahélices de classe III? La dimérisation est-elle simplement un moyen de co-localiser deux récepteurs fonctionnellement indépendants au sein d'un micro-environnement membranaire ou bien faut-il, au contraire, voir un dimère de récepteur heptahélice comme l'unité fonctionnelle d'un RCPG?

Dans le cas du récepteur $G A B A_{B}$, la dimérisation est nécessaire à l'activité du récepteur en permettant une complémentation fonctionnelle entre les récepteurs heptahélices $G_{B B A_{B 1}}$ et $G A B A_{B 2}$. En effet, le domaine Venus flytrap de la sous-unité $G A B A_{B 1}$ contient le site de liaison des agonistes tandis que le domaine heptahélice de la sous-unité $\mathrm{GABA}_{\mathrm{B} 2}$

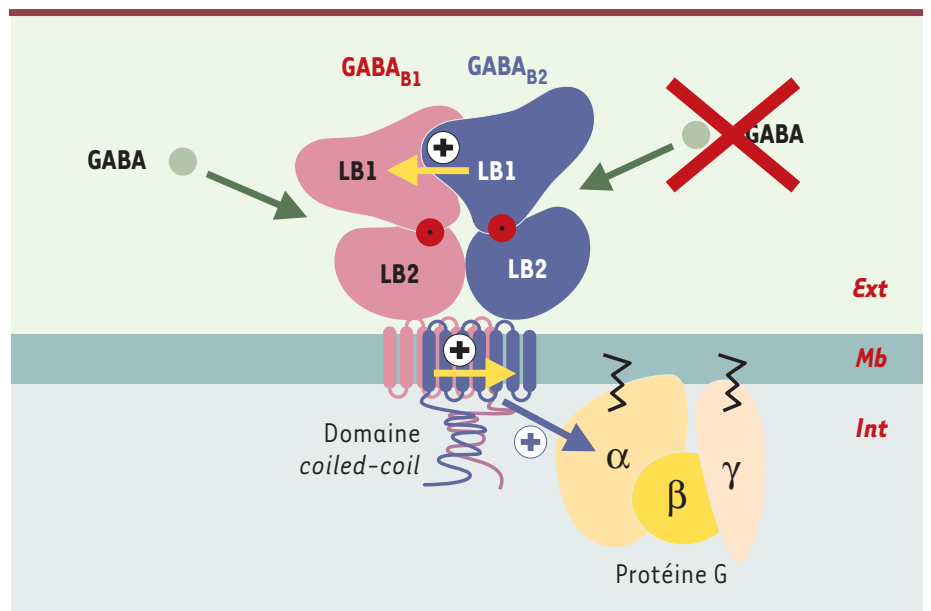

Figure 3. Le récepteur hétérodimérique $G A B A_{B}$. Il est constitué de deux sous-unités $\mathrm{GABA}_{B 1}$ (en rose) et $\mathrm{GABA}_{B 2}$ (en bleu) qui interagissent physiquement, notamment par un domaine coiled-coil intracellulaire. Le domaine Venus flytrap de $G_{B B A_{B 1}}$ constitue le site de liaison de l'agoniste (GABA) sur l'hétérodimère (à gauche). La sous-unité $G_{A B A_{B 2}}$ possède également un domaine semblable, mais qui est incapable de se lier directement au GABA (à droite). Sa présence confère toutefois à $G A B A_{B 1}$ une meilleure affinité pour les agonistes $(\leftarrow+)$. De la même façon, le domaine heptahélice de $G_{A B A_{B 2}}$ contient tous les déterminants nécessaires pour activer les protéines $G$ (sous-unités $\alpha, \beta$ et $\gamma$ ), mais la présence du domaine heptahélice de $\mathrm{GABA}_{B 1}$ est nécessaire pour un couplage optimal $(\rightarrow+)$. Ext: extracellulaire; Int: intracellulaire; $\mathrm{Mb}$ : membrane cellulaire. 
Figure 4. Importance de la dimérisation dans le mécanisme d'activation du récepteur $m \mathrm{Glu}_{1^{*}}$ A. En haut, est représentée la structure tridimensionnelle du dimère de domaines Venus flytrap dans l'état inactif (pdb: lewt, à gauche) et dans l'état actif, en présence de glutamate (pdb: lewk, à droite). Dans les deux cas, l'encadré rouge montre un agrandissement de la zone de contact des lobes 1 (LB1). Cette interface est constituée de deux hélices pour chaque protomère (hélices bleues pour le protomère $A$ et vertes pour le protomère $A^{\prime}$ ). Le passage à l'état activé est caractérisé par un fort changement de l'orientation relative des deux protomères. En bas, le pivotement d'un domaine Venus flytrap par rapport à l'autre est schématisé. Dans ce modèle, la fermeture d'un seul protomère est suffisante pour déclencher le changement conformationnel. 0 : conformation ouverte; $\mathrm{F}$ : conformation fermée. B. Rôle de l'interface de contact des lobes 2 (LB2). Le pivotement au niveau de l'interface des deux lobes I (LB1) illustré en $\boldsymbol{A}$ s'accompagne de la création d'une nouvelle zone de contact entre les deux lobes 2. Ce contact n'est stable, et ne peut donc exister, que si l'un des domaines Venus flytrap est fermé. Les encadrés rouges détaillent les interactions moléculaires ayant lieu à cette interface. La conformation $\mathrm{n}^{\circ} \mathrm{l}$ est un modèle construit en faisant
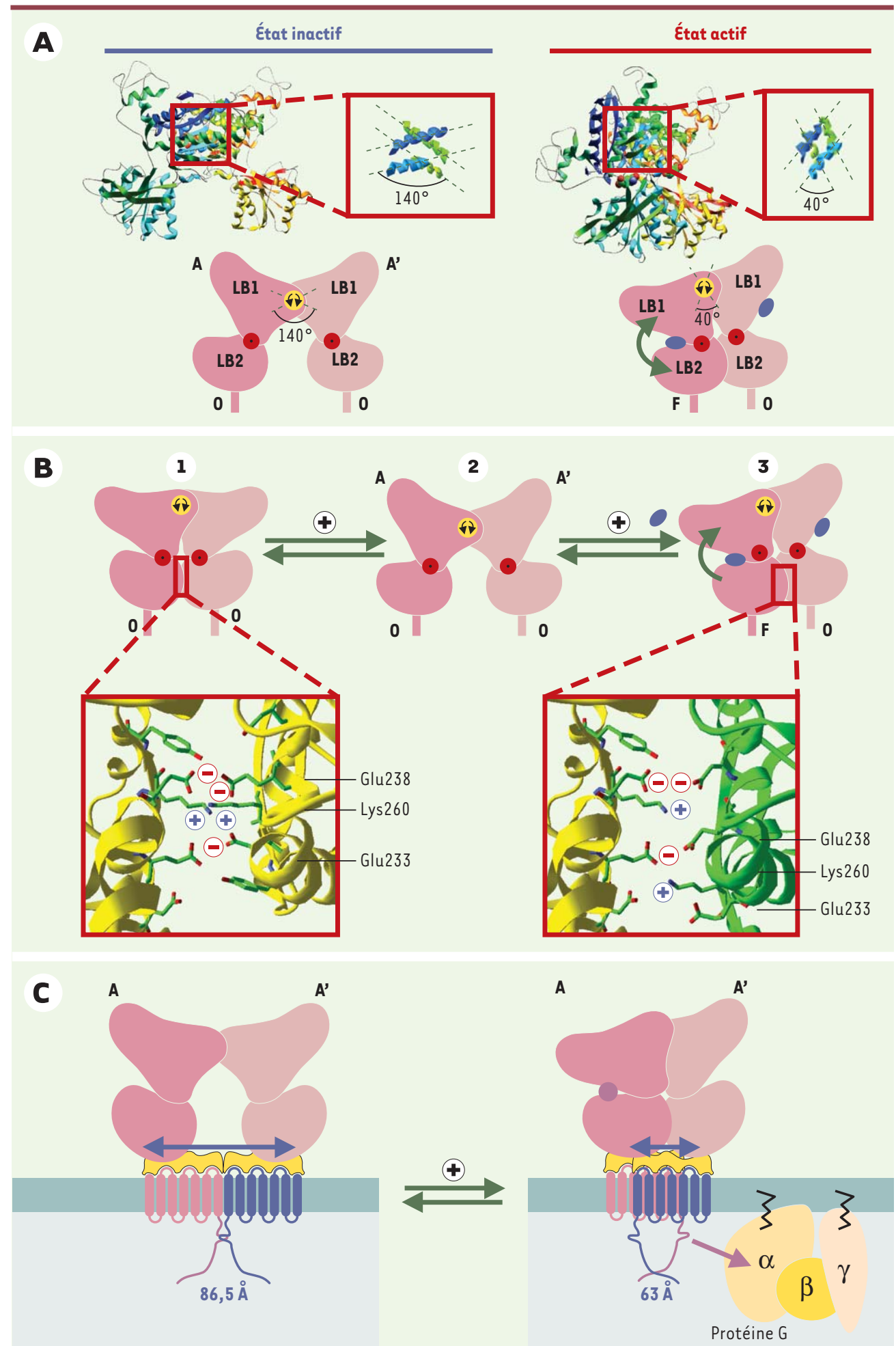

pivoter « manuellement » deux protomères ouverts autour de l'axe de rotation du lobe 1 , de manière à mettre artificiellement en contact les lobes 2. L'interface ainsi créé met face à face des résidus de même charge, ce qui est très défavorable thermodynamiquement et explique pourquoi la probabilité d'existence d'une conformation active réalisée à partir de deux domaines Venus flytrap ouverts est faible. En revanche, dans le cas de figure réel n³, la charge positive de la lysine 260 neutralise en partie la charge du glutamate 238. C. Le pivotement des domaines flytrap a pour conséquence le rapprochement de leurs extrémités carboxy-terminales auxquelles sont rattachés les domaines heptahélices (en bleu) via le domaine riche en cystéines (en jaune). Ce rapprochement pourrait être à l'origine du changement conformationnel des domaines heptahélices, lui-même à l'origine de l'activation des protéines $G$. 
contient les déterminants structuraux permettant le couplage aux protéines $G$. Le domaine heptahélice de $G A B A_{B 1}$ et le domaine Venus flytrap de $G_{A B A_{B 2}}$ jouent également un rôle, puisqu'ils sont nécessaires à une activation optimale du récepteur en augmentant respectivement l'efficacité de couplage aux protéines $G$ et l'affinité des agonistes [22, 23] (Figure 3). Ainsi, les deux types de sous-unités sont non fonctionnels et doivent coopérer pour former un récepteur $\mathrm{GABA}_{B}$ fonctionnel. Ce mode de fonctionnement ne semble pas être unique au sein des récepteurs couplés aux protéines $G$ et pourrait s'appliquer aux récepteurs gustatifs $T_{1}$. En effet, aucun des trois récepteurs heptahélices que sont $T_{1} R 1, T_{1} R 2$ ou $T_{1} R 3$ n'est fonctionnel lorsqu'il est exprimé individuellement, mais l'association des sous-unités $T_{1} R l$ et $T_{1} R 2$ reconstitue un récepteur au « goût sucré », tandis que l'association des sous-unités $T_{1} R l$ et $T_{1} R 3$ est à l'origine $(\rightarrow) \mathrm{m} / \mathrm{s}$ 2000, n० 6-7, p. 852

d'un récepteur du « goût umami » [24, 25] $\rightarrow$.

Un second rôle proposé pour la dimérisation des RCPG de classe III est de permettre la mise en place de mouvements conformationnels de large amplitude entre sousunités, permettant de coupler la fermeture des domaines Venus flytrap extracellulaires à l'activation des domaines heptahélices. Ce mécanisme de type allostérique s'appuie sur la résolution des structures cristallographiques du dimère de domaines externes $d u$ récepteur $\mathrm{mGlu}_{1}$ en présence ou en l'absence d'agoniste ou d'antagoniste. Outre la confirmation du mécanisme de Venus flytrap, ces structures montrent que la liaison d'un agoniste est à l'origine d'un changement conformationnel aboutissant à un changement de l'orientation relative des deux protomères. Deux hélices $\alpha$ parallèles du lobe 1 ( $L B 1$ ) de chaque monomère forment une première zone de contact entre les sous-unités. À l'état inactif, c'est-à-dire en l'absence d'agoniste ou en présence d'antagoniste, l'angle entre les deux couples d'hélices de chaque monomère est de $140^{\circ}$ [9] (Figure $4 A$, à gauche). Les lobes 2 (LB2) sont alors maintenus éloignés par répulsion électrostatique [11] (Figure 4B, conformation $n^{\circ} 2$ ). Lorsque la liaison du glutamate stabilise au moins l'une des deux sous-unités en conformation fermée, les deux domaines extracellulaires pivotent l'un par rapport à l'autre et l'angle des deux couples d'hélices formant l'interface de LBl passe de $140^{\circ}$ à $40^{\circ}$ (Figure $4 \mathrm{~A}$, à droite). Une nouvelle interface de contact est alors créée entre les domaines LB2, stabilisant la conformation active (Figure 4B, conformation $n^{\circ} 3$ ).

\section{Conclusions}

L'ensemble de ces résultats montrent que le changement de conformation d'un domaine Venus flytrap résultant de la fixation d'un agoniste peut être largement ampli- fié grâce à la structure dimérique du récepteur. Comment le pivotement des domaines Venus flytrap se traduit-il par l'activation des domaines heptahélices eux-mêmes responsables de l'activation des protéines G? II n'y a pour le moment pas de réponse, mais il est tentant de supposer que le mouvement relatif de la région extracellulaire repositionne les domaines heptahélices auxquels ils sont rattachés, favorisant ainsi une conformation active de ces derniers (Figure 4C). Un tel mécanisme d'activation n'est pas sans rappeler celui d'autres catégories de récepteurs telles que le récepteur de l'érythropoïétine, ou encore les récepteurs guanylate-cyclase des peptides natriurétiques $[26,27]$. En effet, ces récepteurs existent sous forme de dimères constitutifs, et la fixation de l'agoniste se traduit par une nouvelle conformation de la partie extracellulaire du dimère conduisant à un rapprochement des parties intracellulaires possédant (ou étant associées à) une activité enzymatique.

Il semble donc que des structures dimériques jouent un rôle beaucoup plus général qu'on ne l'imaginait pour permettre le passage d'une information extracellulaire à l'intérieur de la cellule. Ce rôle essentiel de la dimérisation pour l'activation des récepteurs de classe III suggère que les récepteurs heptahélices des classes I et II pourraient également fonctionner sous forme de dimères. En accord avec cette hypothèse, de nombreux récepteurs heptahélices ont été observés sous forme de dimères, mais l'importance de ce phénomène dans le fonctionnement de ces protéines reste une énigme [28]. En quoi une structure dimérique serait-elle importante pour l'activation d'une protéine $G$ hétérotrimérique? En fait, la surface de la protéine $G$ susceptible d'entrer en contact avec un récepteur est à peu près deux fois supérieure à celle de la partie intracellulaire d'un domaine heptahélice [29]. Pourtant, de nombreuses observations montrent qu'un RCPG peut entrer en contact aussi bien avec la sous-unité $\alpha$ qu'avec le dimère $\beta \gamma$ de la protéine $G$. Il est donc possible que seul un dimère de domaines heptahélices ait la capacité d'entrer en contact avec les différents points de la protéine $G$, contact nécessaire à son activation [30]. Cette hypothèse est encore débattue, et d'autres études devront la démontrer ou l'infirmer. $\diamond$

\section{REMERCIEMENTS}

Nous tenons à remercier Francine Acher, Anne-Sophie Bessis, Béatrice Duthey, Julie Kniazeff, Gilles Labesse, Gérald Milioti, Marie-Laure Parmentier, Laurent Prézeau et Philippe Rondard pour les nombreuses discussions stimulantes que nous avons eues, ainsi que Charlotte Deleuze pour la relecture du manuscrit. 


\section{SUMMARY}

How does a $\mathrm{G}$ protein-coupled receptor work?

New insights from the metabotropic glutamate and

$\mathrm{GABA}$ receptors

G-protein coupled receptors (GPCRs) represent the largest membrane proteins family in animal genomes. Being the receptors for most hormones and neurotransmitters, these proteins play a central role in intercellular communication. GPCRs can be classified into several groups based on the sequence similarity of their common structural feature: the heptahelical domain. The metabotropic receptors for the main neurotransmitters glutamate and $\gamma$-aminobutyric acid (GABA) belong to the class III of GPCRs, together with others receptors for $\mathrm{Ca}^{2+}$, for sweet and amino acid taste compounds and for some pheromones, as well as for odorants in fish. Besides their transmembrane heptahelical domain responsible for $\mathrm{G}$-protein activation, most of class III receptors possess a large extracellular domain responsible for ligand recognition. The recent resolution of the structure of this binding domain of one of these receptors, the mGlul receptor, together with the recent demonstration that these receptors are dimers, revealed an original mechanism of activation for these GPCRs. Such data open new possibilities to develop drugs aimed at modulating these receptors, and raised a number of interesting questions on the activation mechanism of other GPCRs. $\diamond$

\section{RÉFÉRENCES}

1. Bockaert J, Pin JP. Utiliser un récepteur couplé aux protéines $G$ pour communiquer: un succès évolutif. CR Acad Sci Ser III 1998; 321: 529-51.

2. Hall RA, Premont RT, Lefkowitz RJ. Heptahelical receptor signaling: beyond the $\mathrm{G}$ protein paradigm. J Cell Biol 1999; 145: 927-32.

3. Palczewski K, Kumasaka T, Hori T, et al. Crystal structure of rhodopsin: A $G$ protein-coupled receptor. Science 2000; 289: 739-45.

4. Josefsson LG. Evidence for kinship between diverse $G$ protein coupled receptors. Gene 1999; 239: 333-40.

5. Bockaert J, Claeysen S, Becamel C, et al. G proteincoupled receptors: dominant players in cell-cell communication. Int Rev Cytol 2002; 212: 63-132.

6. Pin J, Galvez T, Prezeau L. Evolution, structure and activation mechanism of family 3/C G-protein couples receptors. Pharmacol Ther 2003 (sous presse).

7. Hoon MA, Adler $\varepsilon$, Lindemeier J, et al. Putative mammalian taste receptors: a class of taste-specific GPCRs with distinct topographic selectivity. Cell 1999; 96: 541-51.

8. Dulac C. Sensory coding of pheromone signals in mammals. Curr Opin Neurobiol 2000; 10: 511-8.

9. Kunishima N, Shimada Y, Tsuji Y, et al. Structural basis of glutamate recognition by a dimeric metabotropic glutamate receptor. Nature $2000 ; 407$ : 971-7.

10. Tam R, Saier MH Jr. Structural, functional, and evolutionary relationships among extracellular solutebinding receptors of bacteria. Microbiol Rev 1993; 57: 320-46.

11. Tsuchiya D, Kunishima N, Kamiya N, et al. Structural views of the ligand-binding cores of a metabotropic glutamate receptor complexed with an antagonist and both glutamate and $\mathrm{Gd}^{+}$. Proc Natl Acad Sci USA 2002; 99 : 2660-5.

12. Bessis AS, Rondard P, Gaven $F$, et al. Closure of the Venus flytrap module of mGlu8 receptor and the activation process: Insights from mutations converting antagonists into agonists. Proc Natl Acad Sci USA 2002 99: 11097-102.

13. Romano C, Yang WL, O'Malley KL. Metabotropic glutamate receptor 5 is a disulfidelinked dimer. J Biol Chem 1996; 271: 28612-6.

14. Bowery NG, Hill DR, Hudson $A L$, et al. Baclofen decreases neurotransmitter release in the mammalian CNS by an action at a novel GABA receptor. Nature 1980; 283 : 92-4.

15. Kaupmann K, Huggel K, Heid $J$, et al. Expression cloning of $\mathrm{GABA}_{B}$ receptors uncovers similarity to metabotropic glutamate receptors. Nature 1997; 386: 239-46.

16. Jones KA, Borowsky B, Tamm J , et al. GABA B receptors function as a heteromeric assembly of the subunits GABA B R1 and GABA B R2. Nature 1998; 396: 674-9.

17. Kaupmann K, Malitschek B, Schuler V, et al. $\mathrm{GABA}_{B}$ receptor subtypes assemble into functional heteromeric complexes. Nature 1998 ; 396: 683-7.

18. White JH, Wise A, Main MJ, et al. Heterodimerisation is required for the formation of a functional $\mathrm{GABA}_{\mathrm{B}}$ receptor. Nature 1998; 396: 679-82

19. Kuner R, Kohr G, Grunewald $\mathrm{S}$, et al. Role of heteromer formation in $\mathrm{GABA}_{B}$ receptor function. Science 1999; 283: 74-7.

20. Ng Gy, Clark J, Coulombe N, et al. Identification of a $\mathrm{GABA}_{B}$ receptor subunit, $\mathrm{gb} 2$, required for functional $\mathrm{GABA}_{B}$ receptor activity. J Biol Chem 1999; 274 : 7607-10.

21. Margeta-Mitrovic M, Jan $\mathrm{YN}_{\mathrm{N}}$ Jan Ly. A trafficking checkpoint controls $G_{B B}$ receptor heterodimerization. Neuron 2000; 27: 97-106.

22. Galvez T, Duthey B, Kniazeff $J$, et al. Allosteric interactions between $\mathrm{GB} 1$ and GB2 subunits are required for optimal $G A B A_{B}$ receptor function. $E M B O J$ 2001; 20: 2152-9.

23. Kniazeff J, Galvez T, Labesse $G$, et al. No ligand binding in the GB2 subunit of the $G A B A_{B}$ receptor is required for activation and allosteric interaction between the subunits. J Neurosci 2002; 22: 7352-61.

24. Nelson G, Hoon MA, Chandrashekar J, et al. Mammalian sweet taste receptors. Cell 2001; 106 : 381-90.

25. Li X, Staszewski L, Xu H, et al. Human receptors for sweet and umami taste. Proc Natl Acad Sci USA 2002 ; 99 4692-6.

26. Wilson IA, Jolliffe LK. The structure, organization, activation and plasticity of the erythropoietin receptor. Curr Opin Struct Biol 1999; 9: 696-704

27. He X, Chow D, Martick MM, et al. Allosteric activation of a spring-loaded natriuretic peptide receptor dimer by hormone. Science 2001; 293: 1657-62.

28. Angers $S$, Salahpour $A$ Bouvier M. Dimerization: an emerging concept for $G$ protein-coupled receptor ontogeny and function. Annu Rev Pharmacol Toxicol 2002; 42: 409-35.

29. Hamm HE. How activated receptors couple to $\mathrm{G}$ proteins. Proc Natl Acad Sci USA 2001; 98: 4819-21.

30. Lichtarge 0, Bourne HR, Cohen FE. Evolutionarily conserved $G$ (alpha beta gamma) binding surfaces support a model of the $G$ protein-receptor complex. Proc Natl Acad Sci USA 1996 93: 7507-11.

\section{TIRÉS À PART}

T. Galvez 